\title{
Proposal of C-core Type Transverse Flux Motor for Ship Propulsion - Increasing Torque Density by Dense Stator Configuration -
}

\author{
Yuta Yamamoto ${ }^{1}$, Takafumi Koseki ${ }^{1}$, and Yasuaki Aoyama ${ }^{2}$ \\ ${ }^{1}$ Department of Electrical Engineering and Information Systems, Graduate School of Engineering, \\ The University of Tokyo, Tokyo, Japan \\ ${ }^{2}$ Hitachi Research Laboratory, Hitachi Ltd., Ibaraki, Japan \\ *corresponding author, E-mail: y_yamamoto@koseki.t.u-tokyo.ac.jp
}

\begin{abstract}
Electric ship propulsion system has been drawing attention as a solution for savings in energy and maintenance costs. The system is mainly composed of motor, converter and gearbox and required for high torque at low speed. In order to achieve high reliability and low maintenance requirement, direct-drive motor without gearbox has been developed. In this situation, transverse flux motors (TFMs) have been proposed to fulfill the low-speed high-torque characteristic of specific requirement in the ship due to suitable for short pole pitch and large number of poles to increase torque output. In this trend, we have proposed Ccore type motors taking advantage of TFMs' structure. In this manuscript, a simple design method based on the magnetic-circuit theory and simple modeling of the motor is proposed to search a design parameter for maximizing torque as a pre-process of numerical study. The method takes into consideration the effects of magnetic leakage flux, magnetic saturation and pole-core combination in accordance with the systematic theory. The simple modeling is conducted based on a dense armature structure in previous axial flux motors (AFMs) applied to the new motor design. The validity of the method is verified by 3-D finite element analysis (FEA) and relative error is at most $20 \%$. The minimalist design is shown to be advantageous for effective use in 3-D FEA. As a detailed design by the FEA, high torque density and low cogging to output ratio can be achieved simultaneously in the proposed machine.
\end{abstract}

\section{Introduction}

Marine transportation has been recognized as fuel-efficient energy-saving conveyance, but due to upsurge of concerns for environmental problems, energy efficiency design index (EEDI) has been determined for new vessels as a regulation including $\mathrm{CO}_{2}$ emission and fuel efficiency [1]. Advance of electric propulsion system in the ship is essential more than ever. The motors are required for some particular demands - high torque at low speed, compactness, high power factor (PF) and high reliability. Transverse flux motor (TFM) has been given high attention due to its low-speed high-torque characteristics [2] and improved reliability by direct-drive system without gearboxes. We have developed C-core type
TFMs. The earlier design achieved low cogging torque however the output torque was low due to their sparse stator configuration [3]. This manuscript will propose a design method to achieve high torque output with a simple model focused on a pair of the C-type armature core and a field permanent magnet (PM) to determine a limited range of the armature mmf for finite element analysis (FEA). A design process using the method is systematically arranged. The cogging torque included in detailed design of the motor is difficult to calculate exactly using theoretical method and it is preferable to make an estimation using FEA in view of the complex flux path and a lot of considerable constraints. We will demonstrate the suitability of the design method through example of real motor design by 3-D FEA and also proposed a motor with high torque density and low cogging.

\section{Modeling for simple torque estimation}

\subsection{Problems in a previous motor}

In the previous motor [3], an 8-pole 9-core combination as shown in Fig. 1. has been applied to reduce cogging torque which leads to output ripple, noise and non-self activation. In conventional design, windings per phase are not wound due to the different direction of armature currents. Thus, a particular structure called tooth-less structure was adopted to achieve the low cogging torque and easy fabrication simultaneously - a middle tooth with opposite direction of current flow, was removed and a concentrated winding was composed of three tooth per phase in same direction of current flow, while keeping relative positions between magnets and stator teeth as shown in Fig. 2. Besides, an idea of skewing was also introduced. Hence, these methods contribute to low cogging torque, but resulted in a sparse stator configuration by the uncommon structure as shown in Fig. 3. and decreased effective magnetic flux and output. High torque required in ship propulsion was not achieved.

\subsection{Basic strategy for high torque}

In TFMs, it is essential to increase the area that stator tooth faces field permanent magnet (PM) in order to achieve high torque, according to the principle of generated torque in AFMs including TFMs. Conventional AFMs have a dense stator configuration using trapezoidal field PMs, teeth and 


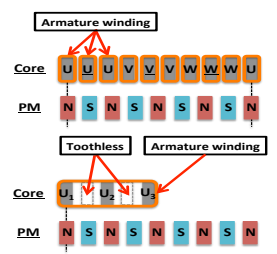

Figure 1: Ordinary 8-pole 9-stator-core combination.

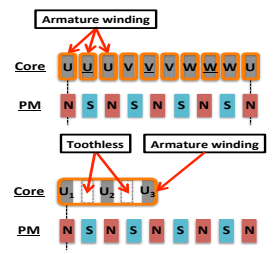

Figure 2: Tooth-less structure applied in the previous motor.

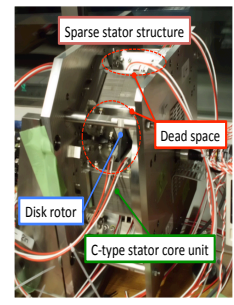

Figure 3: Structure of the previous experimental machine.

windings in the stator $[4,5]$. Therefore, the same structure is introduced to our new design for high torque density and efficient space use in the stator. Furthermore, the tooth-less structure is abolished and an ordinary concentrated winding on each stator-core tooth, observed in the general pole-core combination, is used.

\subsection{1-magnet 1-tooth modeling for simple prediction}

The modeling is conducted on the assumption that a C-coretype stator is also applied to new design. This structure has advantage of simple magnetic flux path due to its separated flux path in every phase. Hence, internal voltage and output torque can be predicted easily from no-load linkage flux identified by using a 1-magnet 1-tooth simple modeling.

The modeling is essential to include visibly following two effects in accordance with the principle of generated torque.

\section{Trade-off between magnetic $\varphi$ and electric loadings $A C$}

- Torque is obtained from interaction between field PM and armature electromagnet and is proportional to the product of magnetic $\varphi$ and electric loadings $A C$. However, it is impossible to maximize each quantity separately due to the spatial restriction. Thus, the modeling is essential to include the trade-off between both loadings.

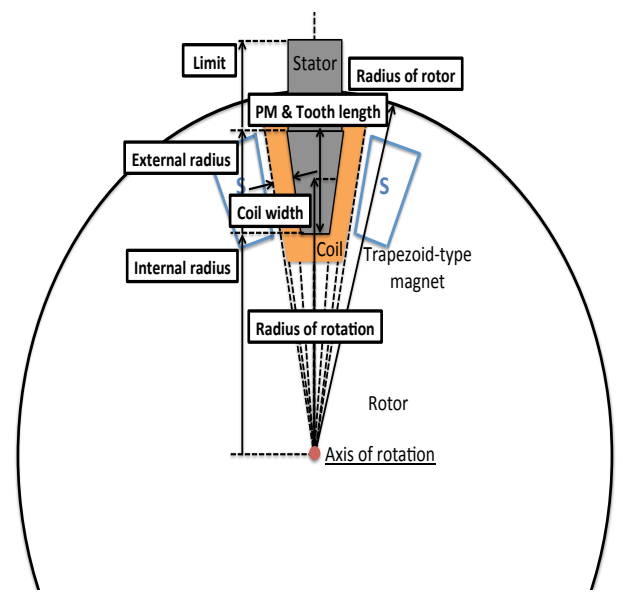

(a) Radial direction component

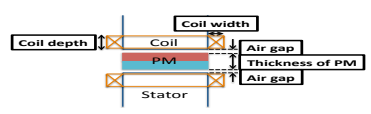

(b) Axial direction component Figure 4: 1-magnet 1-tooth simple modeling.

Table 1: Parameters used for modeling.

\begin{tabular}{ll}
\hline Item & Symbol \\
\hline Number of poles & $2 p$ \\
Number of cores & $n_{c}$ \\
Radius of rotation & $r_{c}$ \\
Radial length of PM & $h_{m}$ \\
Radial length of tooth & $h_{t}$ \\
Thickness of PM & $l_{m}$ \\
Air-gap length & $l_{g}$ \\
Width of PM & $w_{m}$ \\
Width of tooth & $w_{t}$ \\
Area of PM & $S_{m}$ \\
Area of tooth & $S_{t}$ \\
Coil width & $w_{c}$ \\
Coil depth & $l_{c}$ \\
Coil space factor & $d_{c}$ \\
Current density & $j$ \\
\hline
\end{tabular}

2. Leakage flux $\varphi_{l}$ accompanied by changing core structure and increasing armature magnetomotive force ( $\mathrm{mmf}$ )

- It is necessary to consider the leakage flux as the modification is conducted from tooth-less to dense stator structure and the area of tooth is decreased when the area of winding is increasing.

Taken into consideration the above viewpoint, modeling for both radial- and axial-direction component is conducted as shown in Fig. 4. The armature mmf, in proportional to coil width, is chosen as variable and other parameters used for the modeling are shown in Table 1. The whole volume of the motor and amount of PMs is fixed as same as those of the previous motor. Due to variation of the armature $\mathrm{mmf}$, 
the area of tooth can be also varied. A change of operating point based on trade-off between magnetic and electric loadings can be represented by appropriate modeling.

\section{Simple method for high torque density design}

\subsection{Design process and targets for new motor}

In TFM, unfortunately, 3-D numerical study is essential to estimate accurate behavior because of its 3-D magnetic flux path. For that reason, it takes long time to analyze and it is not possible to verify the validity of the analysis. Hence, a simple method using theoretical approach is proposed to obtain the torque prediction beforehand to design quickly and systematically. This simplified method is composed of magnetic-circuit theory and modeling of the motor. Design of a new motor is conducted in accordance with the process as shown in Fig. 5. The minimal design and efficient use in numerical analysis can be achieved simultaneously using this method. Design targets for new motor are determined as high torque density of over $10 \mathrm{Nm} / l$ at current density of $5 \mathrm{~A} / \mathrm{mm}^{2}$ with natural air-cooling while keeping a cogging torque to torque ratio of less than $3 \%$ as shown in Table 2 .

\subsection{PM-flux estimation by permeance method}

A permeance method, one of the systematic and classical design method in PM-type electric machine, is used to obtain magnetic flux generated from PM (called PM flux). The estimation is conducted in following steps.

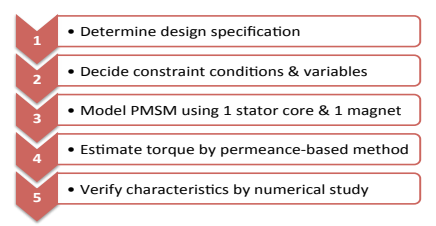

Figure 5: Process of motor design.

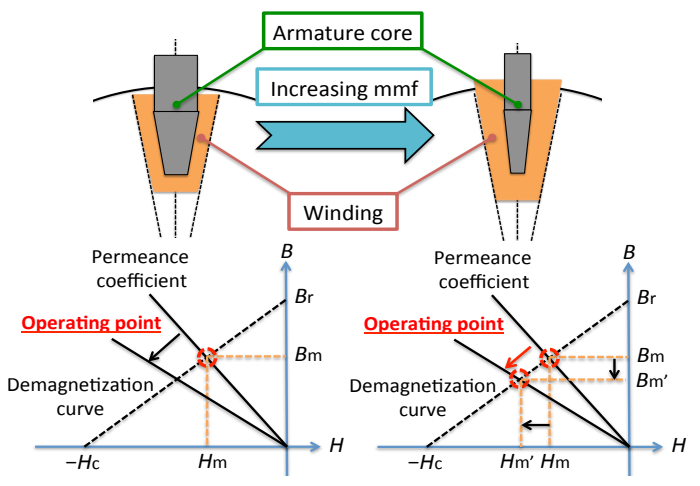

Figure 6: Variation in operating point by armature $\mathrm{mmf}$.
Table 2: Design targets in new motor.

\begin{tabular}{lll}
\hline Item & Symbol & Value \\
\hline Torque density & $\tau$ & $>10 \mathrm{Nm} / l$ \\
Cogging to torque ratio & $\tau_{c o g}$ & $\leq 3 \%$ \\
Rated speed & $N_{r}$ & $<300 \mathrm{rpm}$ \\
\hline
\end{tabular}

A. Determination of a demagnetization curve on PM

- Demagnetization curve is expressed as a linear function using residual flux density $B_{r}$ and coercive force $H_{c}$ of PM as shown in Equation (1).

$$
B(H)=\frac{B_{r}}{H_{c}} H+B_{r}
$$

$B$. Determination of a permeance coefficient $p_{c}$ on PM

- Permeance coefficient $p_{c}$ is expressed as Equation (2) using parameters in Table 1 and Carter coefficient $k_{c}$.

$$
p_{c}=\mu_{0} \frac{S_{t}}{S_{m}} \frac{l_{m}}{k_{c} l_{g}}=-\frac{B_{m}}{H_{m}}
$$

$C$. Determination of operating point $\left(H_{m}, B_{m}\right)$ on PM

- Operating point of PM is defined as intersection point of Equation (1) and (2) as shown in Equation (3).

$$
B_{m}=\frac{B_{r}}{1+\frac{B_{r}}{p_{c} H_{c}}}
$$

\section{$D$. Estimation of PM flux $\varphi_{m}$}

- PM flux is calculated by the product of flux density at operating point and the area of PM as shown in Equation (4). The PM flux corresponds to flux into a stator-core tooth on the assumption that leakage flux does not exist.

$$
\varphi_{m}=B_{m} S_{m}
$$

The area of PM is uniquely determined by given number of poles and amount of magnet, so it is regarded as a constant value. Besides, thickness of PM and air-gap length are also treated as constant. On the other hand, the area of tooth is expressed as a function of armature $\mathrm{mmf}$ (or coil width) as shown in Equation (5).

$$
S_{t}=\frac{2 h_{t}}{\cos \frac{\theta_{c}}{2}}\left\{r_{c} \sin \frac{\theta_{c}}{2}-\frac{3 m m f}{2 j n_{c} d_{c} l_{c}}-\Delta\right\}
$$

where, $\theta_{c}$ and $\Delta$ represent the core-pitch angle and interval between adjacent stator cores.

From the above, the permeance coefficient and the flux are represented as the functions of the armature mmf. Namely, increase/decrease of tooth and winding are accompanied by the variation of armature mmf. The permeance coefficient can be varied according to changing the area of tooth to that of PM ratio. It is contributed to transition of operating point as shown in Fig. 6. The trade-off as referred in Section 2.3. can be described by combination of the permeance method and the modeling. Consequently, they are applied in the following simple and theoretical design method described in Section $\mathbf{3 . 3}$ to $\mathbf{3 . 5}$. 


\subsection{Estimation of effective flux focused on leakage flux}

In Section 3.2., the estimation method has been mentioned without considering decreasing effective flux due to leakage flux. In the real magnetic circuit, leakage flux and magnetic saturation, which do not contribute to generating torque and are not allowed to increase torque in proportional to stator current, are considerable when increasing the armature mmf. Furthermore, another leakage flux path can be created due to the dense structure applied to the new motor design as described in Section 2.2. Accordingly, it is important to include such effects in the estimation, reducing effective flux into tooth caused by getting electric loading larger. In order to describe effective flux, a leakage coefficient $\sigma$ as defined in Equation (6) is introduced.

$$
\sigma=\frac{\varphi_{m}}{\varphi}=\frac{\Theta_{t} P_{t}}{\Theta_{g} P_{g}}=f\left[1+\frac{P_{l}}{P_{g}}\right]
$$

where, $\Theta_{t}, \Theta_{g}, P_{t}, P_{g}, P_{l}, f$ represent total $\mathrm{mmf}, \mathrm{mmf}$ in air gap, total permeance, gap permeance, leakage permeance and coefficient of mmf loss respectively. The effective flux $\varphi$ is calculated by using Equation (6) to correct PM flux $\varphi_{m}$.

\subsubsection{Leakage flux by assumptive flux path method}

Leakage flux paths can be identified by using an assumptive magnetic flux path method focused on the relative position between magnet and tooth. Namely, magnetic flux paths of an analysis object are assumed and separated into typical and basic flux paths as shown in [6]. Based on the paths, the coefficient is calculated. In the following, a radial length of magnet is assumed to be the same as that of tooth.

Case A: PM width is shorter than tooth width $\left(w_{m} \leq w_{t}\right)$

- The flux paths are composed of three parts, one is main path $P_{m}$, another is fringing path at the edge PM $P_{f}$ and the other is leakage path from the side of PM to the side of core $P_{l}$. The main path indicates a trapezoidal part whose area corresponds to the area of tooth and PM. The fringing paths are expressed as half-cylinder and half-ring paths. The flux leakage paths are also described as the half-ring path.

\section{Case B: Otherwise $\left(w_{m}>w_{t}\right)$}

- The main path in Case B is the same as that in Case A. The fringing paths are composed of half-cylinder and onequarter of cylinder paths. The leakage paths are described as half-ring and one-quarter of ring paths.

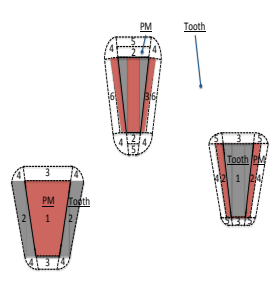

(a) $w_{m} \leq w_{t}$



(b) $w_{m}>w_{t}$
Figure 7: Identification of leakage permeance by using the assumptive magnetic flux path method.

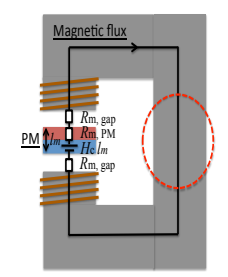

(a) Without consideration

(b) Without consideration

Figure 8: Equivalent expression of magnetic saturation with/without consideration of $\mathrm{mmf}$ loss in the C-type core.

\subsubsection{Magnetic saturation}

A part of tooth relatively decreases compared to that of coil accompanied by increasing armature $\mathrm{mmf}$ as shown in Fig. 6., so leakage flux can be enlarged by a magnetic saturation. The magnetic saturation makes permeability in the C-type core decrease. It can be estimated regarding as a mmf loss in the yoke as well as in the gap as illustrated in Fig. 8. A coefficient of mmf loss, treated as constant value in usual, is defined as the function of the permeability in the stator core in Equation (7). Increase of leakage flux and decrease in the permeability caused by the saturation can be described.

$$
f=\frac{\Theta_{t}}{\Theta_{g}}=\frac{R_{m, g a p}+R_{m, \text { oke }}}{R_{m, g a p}}
$$

where, $R_{m, g a p}$ and $R_{m, y o k e}$ are reluctances in gap and core. Equation (7) is expressed as Equation (8) on the assumption that permeability of PM is the same as that of gap.

$$
f=\frac{\Theta_{t}}{\Theta_{g}}=1+\frac{l_{\text {yoke }}}{l_{\text {gap }}} \frac{\mu_{0} S_{g}}{\mu_{\text {core }} S_{t}}
$$

where, $l_{y}, l_{\text {gap }}, \mu_{0}$ and $\mu_{\text {core }}$ represent the length of yoke and gap including thickness of PM $\left(=l_{m}+l_{g}\right)$, permeability in the space and core respectively. $\mu_{\text {core }}$ is included as appropriate function after conducting approximation from material data.

The coefficient $f$ is also realized as a function of armature mmf because $S_{t}$ is expressed as shown in Equation (5).

The coefficient of $\mathrm{mmf} \operatorname{loss} f$ is determined in three steps.

1. Estimation of tooth flux density taken into consideration the mmf loss only in the C-type core

2. Calculation of permeability $\mu_{\text {core }}$ from $B-H$ curve and the flux density without the saturation.

3. Correction of the coefficient with Equation (8) and $\mu_{\text {core }}$.

Finally, the coefficient of leakage flux $\sigma$ can be derived in accordance with two main ideas described in Section 3.3.1 and 3.3.2.

\subsection{Decrease in effective flux by pole-core combination}

The low cogging torque in a PM-type motor is essential for self-activation, low output ripple, noise and vibration. In the new motor design, a method of pole-slot combination is introduced. This method brings about reducing the effective 
flux due to its difference in relative positions between teeth and magnets as shown in Fig. 1. Hence, the effect is included as a coefficient in estimation of the flux focused on the relative positions as shown in Fig. 9. A basic combination, 4-pole 3-core model, does not exhibit decrease in the effective flux because of its same relative position in each phase. For example, the relative relation between No.5 $\mathrm{PM}$ and No.4 core in a phase $\mathrm{U}$ is the same as the relation numbered 1. On the other hand, 10-pole 9-core combination applied to the new motor has the differences of the relative positions in a same phase between magnets and cores. For instance, in the phase $\mathrm{U}$, the secondary and third cores have phase differences of $\pi / 9 \mathrm{rad}$. and $2 \pi / 9 \mathrm{rad}$. respectively as a basis of the relation between first core and magnet in the phase U.

Thus, the coefficient $k_{p s}$ is simply defined in Equation (9).

$$
k_{p s}=\frac{1}{3}\left(1+\cos \frac{\pi}{9}+\cos \frac{2 \pi}{9}\right) \sim 0.902
$$

This coefficient in a concentrated winding may be treated as winding factor of rotating machine with distributed winding. The flux is estimated based on the simple method using model on behalf of a pole and a C-type core. Thus, the calculation in the pole-combination can be conducted by Equation (9) easily.

\subsection{Derivation of output torque}

An output torque can be derived by the following logically.

$A$. Determination of operating point and PM flux $\varphi_{m}$

$B$. Estimation of no-load effective flux $\varphi$ flowed into tooth

- Calculation of the leakage coefficient including leakage flux and magnetic saturation and pole-core combination can be conducted with Equation (10).

$$
\varphi=k_{p s} \frac{\varphi_{m}}{\sigma}
$$

\section{Calculation of internal voltage and torque}

- An internal voltage in phase can be calculated by using number of turns in each phase $N$ and electrical drive angular velocity $\omega$ as given in Equation (11).

$$
E=N \omega \frac{\varphi}{\sqrt{2}} \frac{n_{c}}{3}
$$

A line current $I$ is calculated with Equation (12).

$$
I=\frac{j S_{c}}{\sqrt{2}}
$$

where, $S_{c}$ represents cross section of conductor. Therefore, a torque $T$ can be identified by conducting quotient of power and mechanical angular velocity.

$$
T=\frac{P}{\omega_{m}}=p \frac{3 E I}{\omega}=\frac{3}{2} p \varphi \cdot m m f
$$

where, $m m f$ shows armature mmf defined in Equation (14).

$$
m m f=\frac{2}{3} n_{c} l_{c} w_{c} d_{c} j
$$

Theoretical torque estimation

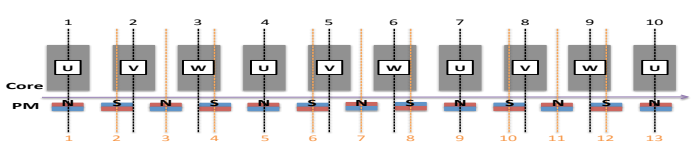

(a) 12-pole 9-core (3 sets of 4-pole 3-core) model

Theoretical torque estimation

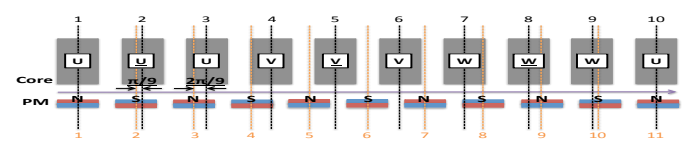

(b) 10-pole 9-core combination model

Figure 9: Relative position between magnets and armature cores in each pole-core combination (Unit: rad.).



Figure 10: Estimation of torque by simple method and FEA.

Equation (13) clarifies that torque is determined by product of magnetic and electric loadings and the assumption in the modeling is reflected into the formula. The torque can be shown graphically by Equation (13). In proportion to the increase of $\mathrm{mmf}$, the flux shall be decreasing accompanied by decrease of area of tooth as shown in Fig. 10. Namely, the tendency of torque indicates monophasic. The width of coil and tooth, important design parameters, can be determined from the value of armature mmf which shows the maximum torque using the proposed method. In the following section, its effectiveness is demonstrated through our motor design.

Table 3: Relative errors between proposed method and FEA

\begin{tabular}{cccc}
\hline $\begin{array}{c}\text { Armature } \mathrm{mmf} \\
{[\mathrm{AT} / \text { phase] }}\end{array}$ & \multicolumn{2}{c}{ Torque $[\mathrm{Nm}]$} & Relative error \\
Proposed & FEA & {$[\%]$} \\
\hline 4800 & 31.1 & 30.3 & 2.8 \\
5500 & 33.7 & 31.0 & 8.7 \\
5900 & 34.2 & 31.0 & 10 \\
6200 & 33.4 & 30.6 & 9.0 \\
\hline
\end{tabular}




\section{Verification of consistency of simple design method and detailed design by $3-D$ numerical study}

\subsection{Validity of simple method against numerical analysis}

White circles as shown in Fig. 9. represent estimated torque by the proposed method. A section of armature mmf, which is output torque of more than $30 \mathrm{Nm}$, is determined for 3-D numerical studies to verify the validity of this method and detailed characteristics and design including cogging torque and consideration of creation. For the section, the 3-D FEAs of static torque are conducted in accordance with the golden section method for optimization and labour saving. The 3-D numerical electromagnetic field analysis is carried out by JMAG, commercial package software using finite element method (FEM). A model with element mesh is illustrated in Fig. 11 and black circles are shown in Fig. 10. Table 3 shows relative errors between the proposed method and FEA. The relative errors are mainly caused by difficulty to estimate flux leakage path especially in large armature mmf. These errors are at most $10 \%$. The good analysis points can be indicated. Therefore, the proposed method is available as a pre-process for the minimalist design and a post-process of FEA for verification of the validity on numerical study.

\subsection{Detailed design and characteristics}

\subsubsection{Determination of design point for detailed design}

The above method is a technique to indicate approximation of torque for detailed design based on FEA. Cogging torque is not predicted. However, in design of PM-type motor with cores, the cogging torque is one of the essential problems contributing to noise, vibration and self activation. It is important to verify the value and reduce to a certain extent. As for estimation, analytical techniques using magnetic energy and conformal mapping have been already published [7-9]. On the other hand, when the shape of motor becomes more complicated and there are a lot of considerable parameters and constraints. Besides, their conditions are interdependent mutually and complexly and it is difficult to include variation in permeance accurately. Consequently, FEA is determined to use for design in detail.

Three points with around maximum torque are chosen and for analysis of cogging torque and the results are shown in Table 4 . The point with lowest peak-to-peak cogging torque to static torque ratio is chosen as immediate design point.

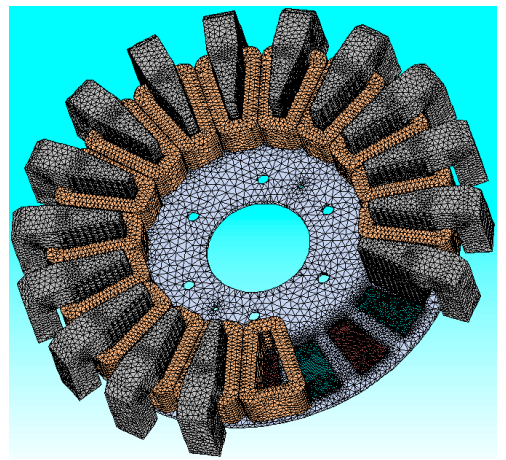

Figure 11: 3-D elements mesh for numerical analysis. (886,736 elements, 238,128 nodes)
Table 4: Peak-to-peak cogging torque to torque ratio

\begin{tabular}{cc}
\hline $\begin{array}{c}\text { Armature mmf } \\
{[\mathrm{AT} / \mathrm{phase}]}\end{array}$ & $\begin{array}{c}\text { Cogging torque to torque ratio } \\
{[\%]}\end{array}$ \\
\hline 5500 & 2.62 \\
5900 & 2.97 \\
6200 & 2.78 \\
\hline
\end{tabular}

\subsubsection{Determination of radius of rotation}

According to [10], it is generally important to adjust the width of magnet and tooth and pole pitch for low cogging torque, but it takes a long time to optimize three parameters by using numerical studies. It is impossible to conduct substantially. Hence, FEAs on both cogging torque and static torque are conducted for radius of rotation on condition that the width of magnet and that of tooth are constant values. The radius of rotation, equivalent to pole pitch, is varied from $60 \mathrm{~mm}$ to $85 \mathrm{~mm}$ at every $2.5 \mathrm{~mm}$. Fig. 12. represents the peak-to-peak cogging torque to torque ratio and the torque density. As a result, the radius of rotation is chosen as $75 \mathrm{~mm}$ due to volume of motor, initial target ratios on both torque density and cogging torque.

\subsubsection{Stator-core design modification for easy creation}

Taking into consideration the construction of the real prototype motor, a trapezoidal stator-core design can be problem depending on its materials. As a material of the core, bulk core, laminated steels and soft magnetic composite (SMC) are applicable.

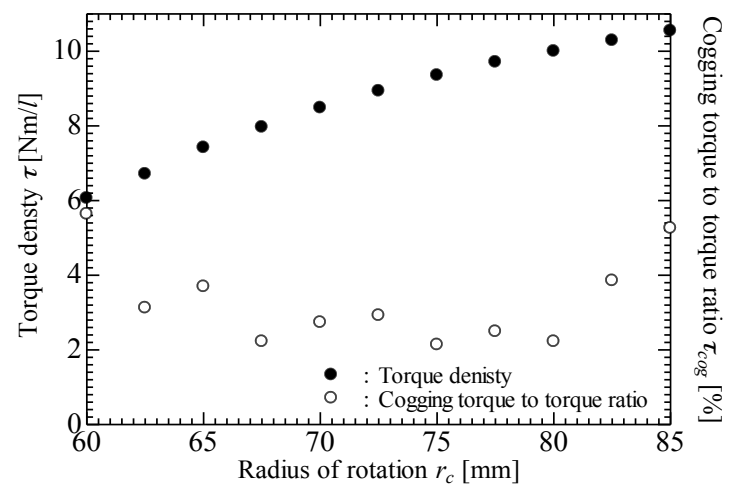

Figure 12: Characteristics of cogging torque to torque ratio and torque density against radius of rotation.

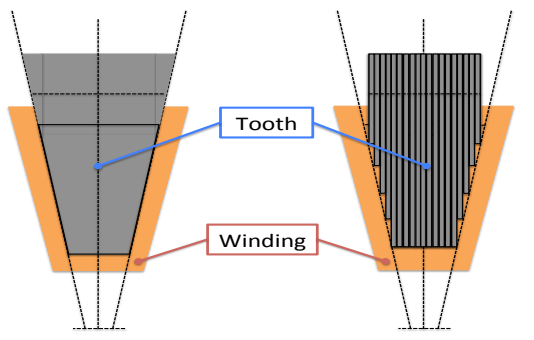

(a) Ideal trapezoidal shape (b) Quasi-trapezoidal shape Figure 13: Stator-tooth structure before/after modification. 
Table 5: Final specification of the prototype motor

\begin{tabular}{lll}
\hline Item & Previous & Proposed \\
\hline Torque density & $5.5 \mathrm{Nm} / l$ & $9.4 \mathrm{Nm} / l$ \\
Cogging to torque ratio & $0.24 \%$ & $1.6 \%$ \\
Rated speed & $84 \mathrm{rpm}$ & $210 \mathrm{rpm}$ \\
Current density & $5 \mathrm{Arms} / \mathrm{mm}^{2}$ & $3.5 \mathrm{Arms} / \mathrm{mm}^{2}$ \\
Area of conductor & $0.28 \mathrm{~mm}^{2}$ & $1.0 \mathrm{~mm}^{2}$ \\
Coil space factor & 0.585 & 0.55 \\
Number of poles & 50 poles & 20 poles \\
Number of turns per unit & 400 turns & 176 turns \\
Power factor & 0.757 & 0.983 \\
\hline
\end{tabular}

The SMC has high flexibility in forming the shape, but it is still high cost and low permeability and saturation magnetic flux density characteristics. Furthermore, the bulk-type core can be also shaped easily, but the eddy current loss will be significant, especially at high speed or harmonics caused by pulse-width-modulation (PWM) drive.

For that reason, the laminated steels have been chosen as a material of stator core. In order to apply them, a quasitrapezoid stepped teeth as shown in Fig. 13. is introduced. A quantitative verification of characteristics is given in Table 5. The possibility to fulfill roughly initial targets in new motor design is verified as a result of the FEA. Besides, high power factor (PF) of 0.983 can be achieved compared to conventional TFMs as shown in $[11,12]$ due to small number of turns, minimization of leakage flux and small number of poles. The high PF enables a whole motor drive system to reduce its volume and contributes to increasing an advantage, flexibility of space layout in the ship, by electric propulsion system.

\section{Conclusion}

This manuscript has introduced the simple design method composed of the simple modeling focused on principle to generate torque and systematic theory of permeance method. The proposed method is applied to search a parameter for high torque as a pre-design process before detailed design by 3-D numerical analysis.

The consistency of the method has been verified through the 3 -D FEA. The relative error is at most $10 \%$. This method is applicable as a pre- and post-process tool of FEA to conduct the minimalist design and verify validity of the numerical computation.

The proposed motor designed by the simple method and the detailed design by FEA is clarified to achieve nearly initial targets, torque density of more than $10 \mathrm{Nm} / l$ and cogging to output torque ratio of less than $3 \%$. In spite of low current density, the torque density of the proposed motor is verified to achieved twice as same as that of the previous machine. In addition, the high power factor of more than 0.90 , which is not achieved in another conventional TFMs, is clarified to realize due to low leakage flux and small number of turns.

A prototype machine based on this manuscript is presently under construction and we are preparing for the experiment to verify the consistency between design and the real motor.

\section{Acknowledgements}

We would like to thank the members of Hitachi Research Laboratory for their technical advice and support of this research and those of JAE Shinshu, MT Drive, ACM and Shin-Etsu Chemical for creating the proposed new motor.

\section{References}

[1] International Maritime Organization (IMO), Emissions from fuel used for international aviation and maritime transport, Subsidiary Body for Scientific and Technical Advice, SBSTA 35, 2011.

[2] H. Weh, H. Hoffman, and J. Landrath, New Permanent Magnet Excited Synchronous Machine with High Efficiency at Low Speeds, Proc. Elect. Mach., ICEM, 1988.

[3] T. Nakamura, T. Koseki, and Y. Aoyama, A low-speed high-torque permanent magnet synchronous motor Reducing cogging torque and eddy current loss -, Journal of JSAEM, Vol. 20, No. 2, pp. 410-415, 2012.

[4] T.J. Woolmer, and M.D. McCulloch, Analysis of the Yokeless and Segmented Armature Machine, IEEE Elect. Mach. Drives Conf., IEMDC, pp. 704-708, 2007.

[5] H. Tokoi, S. Kawamata, and Y. Enomoto, Study of High-Efficiency Motor Using Soft Magnetic Cores, IEEJ Trans. Indus. Appli., Vol. 132, No. 5, pp. 574-580, 2011 (in Japanese).

[6] J.F. Gieras, R.J. Wang, and M.J. Kamper, Axial Flux Permanent Magnet Brushless Machine 2nd Edition, Springer, 2008

[7] Y. Kano, T. Kosaka, and N. Matsui, Study on Optimum Design of Multi-pole Interior Permanent Magnet Motor with Concentrated Windings - Motor Volume Minimization of IPM Motor for Low-Speed, HighTorque Applications -, IEEJ Trans. Indus. Appli., Vol. 127, No. 4, pp. 349-359, 2007 (in Japanese).

[8] M. Markkovic, M. Jufer, and Y. Perriard, Reducing the Cogging Torque in Brushless DC Motors by Using Conformal Mappings, IEEE Trans. Manetics, Vol. 40, No. 2, pp. 451-455, 2004

[9] D. Zarko, D. Ban, and T.A. Lipo, Analytical Solution for Electromagnetic Torque in Surface PermanentMagnet Motors Using Conformal Mapping, IEEE Trans. Magnetics, Vol. 45, No. 7, pp. 2943-2954, 2009

[10] T. Nakamura, J.S. Shin, and T. Koseki, Cogging force reduced by pole-slot combination in a transverse-flux type Permanent Magnet Linear Synchronous Motor, IEE Tech. Meet. Linear Drive, LD-11-030, pp. 41-46, 2011 (in Japanese).

[11] M.R. Harris, G.H. Pajooman, and S.M. Abu Sharkh, The problem of power factor in VRPM (transverse-flux) machines, IEEE Elect. Mach. Drives Conf., IEMDC, pp. 386-390, 1997.

[12] S.M. Husband, and C.G. Hodge, The Rolls-Royce transverse flux motor development, IEEE Elect. Mach. Drives Conf., IEMDC, Vol. 3, pp. 1435-1440, 2003. 